\title{
FoxP3: A Key Player in T Regulatory Biology
}

\author{
D. N. Rao $\cdot$ Raza Ali Naqvi
}

Published online: 4 February 2011

(C) Association of Clinical Biochemists of India 2011

The immune system has adapted mechanisms to recognize and eliminate threats, as well as to defend against selfdestruction. Tolerance to self-antigens and defence against non self pathogens are maintained by the different immune cells. If there is an imbalance in $\mathrm{T}$ reg cells population, may leads to auto immunity. The generation of natural Treg (nTreg)/induced Treg (iTreg) and its maintenance of suppressive function are crucial for immune homeostasis. There are various surface markers on $\mathrm{T}$ cells like CD25, CTLA-4, OX-40 etc. and intracellular marker like FoxP3 involved in the generation and maintenance of suppressive function of Treg cells. Recent work has shown that forkhead DNA-binding proteins FoxP3, is X-chromosomeencoded transcription factor uniquely involved in the development and function of the T-regulatory cells. Lack of FoxP3 leads to development of fatal autoimmune lymphoproliferative disease in humans known as IPEX; (immune dysregulation, polyendocrinopathy, enteropathy, $\mathrm{X}$-linked) furthermore, ectopic FoxP3 expression induces conversion of effector $\mathrm{T}$ cells to regulatory $\mathrm{T}$ cells. The regulation of FoxP3 expression, and its role in maintenance of $\mathrm{CD} 4{ }^{+} \mathrm{CD} 25^{+} / \mathrm{CD} 25^{-}$Treg fitness still a matter of debate.

Being a transcription factor FoxP3 activates/represses various effectors of the $\mathrm{T}$ cell activation/inhibition signal pathways. FoxP3 is an acetylated, oligomeric component of large molecular complex, actively represses transcription by recruiting enzymatic corepressors, including histone modifiers during its function. Chromatin remodeling through FoxP3 associated complexes may dynamically

D. N. Rao $(\varangle) \cdot$ R. A. Naqvi

Department of Biochemistry, All India Institute of Medical Sciences (AIIMS), Ansari Nagar, New Delhi 110029, India e-mail: dnrao311@rediffmail.com affect the multiple immunological signaling networks, eventually in summation lead to immune suppression. FoxP3 interacting proteins (CTLA-4, IL-2, IL-2RA, NFATC2 etc.) complex plays a central role in inducing gene transcription during the immune response. Transcription factors function as a carrier to take histone modifiers at particular locus at which they impose their effect. FoxP3 interacting with histone acetyl transferases, helps to maintain the acetylated state of FoxP3. Dynamic regulation of HAT and HDAC in FoxP3 acetylation confers the suppressive index of nTreg and iTreg cells.

\section{FoxP3 Directed Treg Functions}

The functional requirement of FoxP3 for establishment and maintenance of Treg specific suppressor function may not indicate the development of fatal rapidly progression disease in FoxP3 mutant mice and human developed due to Treg deficiency, it might develop due to some other pathogen invasion. Dramatic enhancement of FoxP3 expression and acquisition of Treg phenotype by conventional/activated $\mathrm{T}$ cells in different pathogenic condition. Recently observations in mice, indicating that genetic marking of FoxP3 protein is mostly limited to $\mathrm{T}$ cells. Although the predominant $\mathrm{FoxP}^{+}$expression present in $\mathrm{CD}^{+}$thymocyte and peripheral $\mathrm{T}$ cell populations, however small numbers of $\mathrm{CD}^{+}$, double positive and double negative thymocyte and peripheral $\mathrm{T}$ cell subsets also express FoxP3. Some existing experiments in wild-type mice also indicates that FoxP3 is expressed primarily in a subset of $\mathrm{CD}^{+}{ }^{+} \mathrm{T}$ cells and display a diverse TCR repertoire. The FoxP $3{ }^{+} \mathrm{CD} 25^{+} \mathrm{CD} 4^{+} \mathrm{T}$ cells/CD25 $5^{-}$show suppressive function, but it is distinct from conventional naive $\mathrm{CD}_{25}{ }^{-} \mathrm{FoxP}^{-}$and activated $\mathrm{CD} 25^{+} \mathrm{FoxP}^{-} \mathrm{T}$ cells. 
However, the FoxP3 deficiency within the T cells leads to development of fatal disease in FoxP3 mutant mice. This result raised the important question whether transient upregulation/down regulation of FoxP3 has a cell-intrinsic role in deletion of or anergy induction in self-reactive thymocytes and peripheral $\mathrm{T}$ cells, or in the regulation of peripheral $\mathrm{T}$ cell responses. Further studies on FoxP3 ${ }^{-}$and wild-type bone-marrow transfers into irradiated or $\mathrm{T}$ celldeficient hosts, in presence or absence of transgene-encoded cognate antigens. This study confirms that FoxP3 is essential for generation of Treg. Furthermore the development of Treg paucity, resulting of FoxP3 deficiency and subsequent development of fatal autoimmune lesions in mice and humans. Taken together, this study firmly confirms the FoxP3 expression is essential for Treg generation and maintaining its dedicated lineage of suppressor $\mathrm{T}$ cells.

\section{FoxP3 and Immune Regulatory Network}

Ectopic expression of FoxP3 in activated and conventional $\mathrm{T}$ cells confer its suppressive activity, by the upregulation of Treg associated molecules such as CD25, cytotoxic T-lymphocyte-associated antigen-4, and glucocorticoidinduced TNF-receptor-family-related protein. However, the mechanism by which FoxP3 controls these molecular events has yet to be explained. The peripheral Treg differentiation in the thymus started upon induction of FoxP3 expression in a subset of thymocyte expressing $\alpha \beta$-TCRs. A stronger TCR engagement and CD28 costimulatory receptor facilitate FoxP3-dependent regulatory $\mathrm{T}$ cells differentiation. FoxP3 responsive gene expression profiling further support the above discussed observations that a large number of genes involved in development and function of Treg like Il2ra, Ctla4, Tnfsrl8 etc. whose change of expression was less in $\mathrm{GFP}^{+} \mathrm{FoxP}^{-} \mathrm{T}$ cells as compared to wild-type FoxP $3^{+} \mathrm{T}$ cells. If some of these genes are upregulated/downregulated upon FoxP3 expression in activated or conventional $\mathrm{T}$ cells, as transient fashion or to a lesser degree, further confers that FoxP3 is involved in the maintenance of Treg suppressor functions. Thus, the ultimate upshot of FoxP3-dependent transcriptional program may modulate the adaptive/innate characteristics, like beneficial, adjusting, disadvantageous and potentially dangerous for establishment of Th1-Th2-Th17 features. Some recent studies have also suggested that association of miRNA toward regulation of FoxP3 expression and development of Treg cells. An miR signature has been explored in the human and mouse $\mathrm{T}$ regs. FoxP3 induced miR-155 actively involved in the generation of $\mathrm{T}$ reg cells, however some other miR signature like miR-31 and miR-21 involved in the regulation of FoxP3 expression.

\section{Bibliography}

1. Zheng Y, Rudensky AY. FoxP3 in control of the regulatory T cell lineage. Nat Immunol. 2007;8(5):457-62. Review.

2. Ono M, Yaguchi H, Ohkura N, Kitabayashi I, Nagamura Y, Nomura T, Miyachi Y, Tsukada T, Sakaguchi S. FoxP3 controls regulatory $\mathrm{T}$-cell function by interacting with AML1/Runx1. Nature. 2007;446:685-9.

3. Marson A, Kretschmer K, Frampton GM, Jacobsen ES, Polansky JK, MacIsaac KD, Levine SS, Fraenkel E, von Boehmer H, Young RA. FoxP3 occupancy and regulation of key target genes during T-cell stimulation. Nature. 2007;445:931-5.

4. Kumar S, Ali Naqvi R, Khanna N, Rao DN. Disruption of HLA-Dr raft, deregulations of Lck-ZAP-70-cbl-b Switches and miR-181a towards $\mathrm{T}$ cell hyporesponsiveness in leprosy. Plos one. 2010 (under review).

5. Kumar S, Ali Naqvi R, Khanna N, Rao DN. FoxP3 $\mathrm{T}$ cells instrumental to mediate immune suppression with the progression of leprosy. Nat Med. 2010 (under review).

6. Belkaid Y, Rouse BT. Natural regulatory $\mathrm{T}$ cells in infectious disease. Nat Immunol. 2005;6:353-60.

7. Li B, Greene MI. FoxP3 actively represses transcription by recruiting the HAT/HDAC complex. Cell Cycle. 2007;6:1432-6.

8. Rouas R, Fayyad-Kazan H, El Zein N, Lewalle P, Rothé F, Simion A, Akl H, Mourtada M, El Rifai M, Burny A, Romero P, Martiat P, Badran B. Human natural Treg microRNA signature: role of microRNA-31 and microRNA-21 in FoxP3 expression. Eur $\mathbf{J}$ Immunol. 2009;39:1608-18. 